\title{
Anticoagulation Management
}

\author{
Blaze W. Cook, M.D. ${ }^{1}$
}

\section{ABSTRACT}

To perform safe invasive procedures on patients on anticoagulation therapy, management of the patient's anticoagulation status is mandatory. For patients on anticoagulation therapy, a risk analysis of both the procedural risk of bleeding as well as the risk to the patient from discontinuing anticoagulation needs to be performed. For procedures at low risk of bleeding, the procedure may be performed with little change to the patient's anticoagulation regimen. For procedures with significant bleeding risk, however, anticoagulation will need to be held or reversed. Patients at high risk for thromboembolic complications will require bridging therapy if anticoagulation is to be withheld. In this article, a logical assessment of bleeding risk in patients undergoing procedures is presented, and a review of anticoagulation and risk is discussed.

KEYWORDS: Anticoagulation, heparin, warfarin, bleeding

Objectives: Upon completion of this article, the reader should be able to explain the management of anticoagulant medications and be able to perform a risk assessment of anticoagulation cessation.

Accreditation: Tufts University School of Medicine (TUSM) is accredited by the Accreditation Council for Continuing Medical Education to provide continuing medical education for physicians.

Credit: Tufts University School of Medicine designates this journal-based CME activity for a maximum of 1 AMA PRA Category 1 Credit $^{\mathrm{TM}}$. Physicians should claim only the credit commensurate with the extent of their participation in the activity.

Medical management of patients on anticoagulation is an integral part of safely performing invasive procedures. Warfarin (Coumadin $^{\circledR}$, Bristol-Myers Squibb Co., Princeton, NJ) is the most widely used oral anticoagulation medication, and the indications for its use vary. Heparin and low-molecular-weight heparins are the most common parenteral anticoagulation medications. Although less common, other anticoagulation formulations exist. In this article, the author discusses the periprocedural management of anticoagulation and the role of bridging therapy for patients on long-term anticoagulation; antiplatelet agents are discussed elsewhere in this issue. The agents themselves are discussed, followed by an examination of procedural and patient risk.

\section{ANTICOAGULATION AGENTS (Table 1)}

\section{Heparin}

Heparin is supplied in two general formulations. Unfractionated heparin (UFH) is a heterogenous mixture of glycosaminoglycans with a range of molecular weights from 3,000 to 30,000 daltons (Da; mean of $15,000 \mathrm{Da}$; generally only one-third of the molecules will be biologically active. ${ }^{1,2}$ Low-molecular-weight heparin
${ }^{1}$ Department of Radiology, University of Colorado Health Sciences Center, Denver, Colorado.

Address for correspondence and reprint requests: Blaze W. Cook, M.D., Denver Health Medical Center, Radiology Specialists of Denver, 777 Bannock Street, Denver, CO 80204-4597 (e-mail: blaze@blazecook.com).

Pharmacology in Interventional Radiology; Guest Editors, Kimi L.
Kondo, D.O. and Charles E. Ray, Jr., M.D.

Semin Intervent Radiol 2010;27:360-367. Copyright (C) 2010 by Thieme Medical Publishers, Inc., 333 Seventh Avenue, New York, NY 10001, USA. Tel: +1(212) 584-4662.

DOI: http://dx.doi.org/10.1055/s-0030-1267849.

ISSN 0739-9529. 
Table 1 Pharmacology of Commonly Used Anticoagulants*

\begin{tabular}{|c|c|c|c|}
\hline Agent & Half-Life & $\begin{array}{l}\text { Monitoring } \\
\text { Serum Test }\end{array}$ & Reversal Agent \\
\hline Warfarin (Coumadin ${ }^{\circledR}$ ) & 40 hours & INR & Fresh frozen plasma \\
\hline Unfractionated heparin & 1.5 hours & aPTT, ACT & Protamine (1 mg/100 IU) \\
\hline LMWH: Enoxaparin (Lovenox ${ }^{\mathbb{R}}$ ) & 4.5-7 hours & Anti-Xa & $\begin{array}{l}\text { Incomplete. Protamine ( } 1 \mathrm{mg} / 1 \mathrm{mg} \text { enoxaparin); } \\
\text { repeat at half dose if needed. }\end{array}$ \\
\hline LMWH: Dalteparin (Fragmin ${ }^{\mathbb{R}}$ ) & $2-5$ hours & Anti-Xa & $\begin{array}{l}\text { Incomplete. Protamine ( } 1 \mathrm{mg} / 100 \mathrm{IU}) \\
\text { repeat at half dose if needed. }\end{array}$ \\
\hline LMWH: Tinzaparin (INNOHEP $\left.{ }^{\circledR}\right)$ & $3-4$ hours & Anti-Xa & $\begin{array}{l}\text { Incomplete. Protamine }(1 \mathrm{mg} / 100 \mathrm{IU}) \\
\text { repeat at half dose if needed. }\end{array}$ \\
\hline Fondaparinux (Arixtra ${ }^{\circledR}$ ) & 17 hours & Anti-Xa & None. Consider recombinant factor VIla. \\
\hline Bivalirudin (Angiomax ${ }^{\circledR}$ ) & 25 minutes & aPTT & None. Consider recombinant factor VIla. \\
\hline Argatroban & 45 minutes & aPTT & None. Consider recombinant factor VIla. \\
\hline
\end{tabular}

*In consideration of pre/periprocedural anticoagulation, elimination of the agent is of primary concern. In general, five half-lives are required for complete elimination of a substance. If the procedure must be performed before that time frame, reversal agents are listed for reference. INR, international normalized ratio; aPTT, activated partial thromboplastin time; ACT, activated clotting time; LMWH, low-molecular-weight heparin.

Adapted from Hirsh et al1.

(LMWH) has a molecular weight of 2,000 to 9,000 Da (mean of 4,000 to 5,000 Da). The smaller fragments of LMWH do not bind well to plasma proteins, macrophages, and endothelial cells, allowing LMWH to have a more predictable effect than UFH, which binds variably. ${ }^{2}$ Both UFH and LMWH complexes with antithrombin (formerly called antithrombin III), converting antithrombin from a slow to a rapid inactivator of thrombin and other factors including $\mathrm{Xa}^{1}{ }^{1}$ Once antithrombin complexes with thrombin, heparin dissociates and returns to circulation. Short-chain heparins (which comprise a larger percentage of heparin molecules in LMWH as compared with UFH) cannot bridge antithrombin and thrombin, and mediate their effects by the inhibition of factor Xa. By inhibiting thrombin, heparin also inhibits platelet aggregation and activation of other clotting factors. ${ }^{1}$

Heparin is not absorbed orally and therefore must be administered parenterally. The two preferred routes of administration are intervenous (IV) and subcutaneous, with reduced bioavailability by the subcutaneous route. After entering the bloodstream, heparin binds to plasma and cellular proteins, reducing its effect. ${ }^{1} \mathrm{Hep}^{-}$ arin is cleared with a half life of 60 to 90 minutes in a nonlinear fashion by both rapid and slow mechanisms. The rapid and saturable mechanism of heparin clearance is primarily due to the binding of cellular receptors, where it is internalized and degraded. ${ }^{1}$ The slower and nonsaturable mechanism is largely renal and accounts for a disproportionate amount of clearance at high doses, leading to the observation that as plasma concentrations of heparin increase, the rate of clearance decreases. ${ }^{1}$ As an example, assuming a $70 \mathrm{~kg}$ adult, a 7,000 unit IV heparin dose will have a half-life of 60 minutes whereas an 1,800 unit dose would have a half-life of 30 minutes. A large dose of 28,000 units would have a half-life of 2 hours and 30 minutes. For practical purposes, the effective half-life of heparin is 60 to 90 minutes. ${ }^{1}$

A typical initiating dose of full-dose UFH for therapeutic purposes is either empiric or weight based. An empiric dose is a 5,000-unit IV bolus followed by a continuous infusion of $1,000 \mathrm{U} / \mathrm{h} \mathrm{IV}$. A weight-based dosing regimen with a bolus of $80 \mathrm{U} / \mathrm{kg}$, followed by a continuous $18 \mathrm{U} / \mathrm{kg} / \mathrm{h}$ infusion was found to be more effective in preventing recurrent thromboembolism when compared with nonweight-based regimens. ${ }^{3} \mathrm{Re}-$ duced doses are used in acute coronary syndromes and in conjunction with fibrinolytic agents.

The activity of unfractionated heparin is most commonly monitored by the serum-activated partial thromboplastin time (aPTT). ${ }^{1,4}$ For full anticoagulation, the goal is aPTT in the range of 1.5 to 2.5 times normal. It should be noted that an aPTT effects of heparin are nonlinear, and at higher concentrations of circulating heparin, the aPTT result becomes less accurate. ${ }^{4}$ The activated clotting time (ACT) is an assessment of the time it takes whole blood to clot in the presence of an activator. ${ }^{5}$ The ACT is useful to monitor procedural anticoagulation during invasive procedures because it has a linear relationship with heparin and does not exhibit the variability at high doses that is seen with the aPTT. The ACT also has the added advantage of being an assessment of overall coagulation ability. The ACT monitoring regimen is similar to the prolongation of the aPTT, with an ACT of 1.5 to 2.5 times baseline in a fully anticoagulated patient (or $>200$ seconds if no baseline was performed; normal is $<150$ seconds).,

Rapid reversal is a major advantage to the use of heparin. Aside from the relatively short half-life of UFH as compared with other anticoagulation agents, heparin may be fully reversed pharmacologically. Protamine is a protein derived from fish sperm with a half-life of 
7 minutes that binds heparin to form an inert salt, which is then degraded and does not induce a hypercoagulable state. ${ }^{1}$ One mg of protamine will neutralize $~ 100$ units of heparin. Two considerations need to be made when considering protamine reversal of heparin. First, given the short half-life of heparin, only the past hour or two of heparin administration needs to be considered for reversal. As an example, for a patient on a continuous drip of heparin at 1000 units/hour, less than $30 \mathrm{mg}$ of protamine is required for complete reversal. Second, if subcutaneous heparin was administered, the slow elution to the bloodstream will likely continue after the relatively rapid clearance of protamine. The most common side effects of protamine are bradycardia and hypotension, which can be avoided by slow administration. ${ }^{1}$

There are two major nonhemorrhagic complications of heparin, heparin-induced thrombocytopenia (HIT) and osteopenia. HIT occurs in $\sim 4$ to $5 \%$ of patients given heparin and typically develops between days 5 and 14 of continuous therapy. ${ }^{6}$ Heparin-induced osteoporosis is a consideration for patients on long-term heparin therapy. Although the true incidence of clinically significant osteoporosis (resulting in fractures) is not known, it may be as high as 15\%. Clinically insignificant osteoporosis (detectable osteoporosis) is much more common and may be as high as $40 \%{ }^{7}$

\section{LOW-MOLECULAR-WEIGHT HEPARIN}

Low-molecular-weight heparin (LMWH; including enoxaparin, tinzaparin, dalteparin, and nadroparin) is derived from unfractionated heparin by various means and the variability in derivation accounts for the multiple commercially available forms. Although there are specific differences between forms, the general pharmacology is similar within the class. All LMWH are formulated to a weight of one-third of UFH, which reduces binding to proteins and cells. Additionally, the crosslinking of antithrombin and thrombin is reduced, as the relative number of long chains required for bridging is decreased. These two factors explain the pharmacologic differences between LMWH and UFH.

The reduced binding of plasma and cellular proteins makes the dose-response relationship more predictable with LMWH, and results in a half-life of 2 to 4 times unfractionated heparin. ${ }^{1}$ Reduced binding of platelet receptors reduces the incidence of heparin-induced thrombocytopenia by two-thirds, and reduced binding to osteoclasts decreases the incidence of osteopenia. However, reduced binding of cellular proteins (macrophages) increases the fraction cleared by the kidneys, becoming unpredictable at a creatinine clearance of $<30 \mathrm{~mL} / \mathrm{min}$, therefore doses should be reduced in patients with renal impairment. ${ }^{1}$

Reduced bridging of antithrombin and thrombin makes the inhibition of other factors more important with regards to the anticoagulant effects of LMWH. As a result, the aPTT underestimates the anticoagulant effect of LMWH and the anti-Xa activity is a more accurate estimate of the anticoagulation due to LMWH. However, due to predictable pharmacokinetics, routine testing is not generally performed. ${ }^{1}$ These predictable pharmacokinetics make LMWH useful in the outpatient setting as well as for bridging warfarin for invasive procedures as it does not require the frequent monitoring that UFH necessitates. This benefit is in addition to the reduced incidence of heparin-induced thrombocytopenia and osteopenia. A disadvantage is an increased cost.

Protamine sulfate is also used to reverse LMWH, although it is not as effective or predictable as when used for the reversal of UFH. Dosage is also different when used to reverse LMWH, with consideration of anti-Xa units, and anti-Xa units are different for the different LMWH formulations. For illustration purposes, $1 \mathrm{mg}$ of enoxaparin is 100 anti-Xa units. Protamine is then dosed at $1 \mathrm{mg}$ per 100 anti-Xa units. A second dose of $0.5 \mathrm{mg}$ protamine sulfate per 100 anti-Xa units should be administered if bleeding continues ${ }^{1}$ because the half-life of protamine is only $\sim 7$ minutes.

\section{Bivalirudin (Angiomax ${ }^{\circledR}$ )}

Bivalirudin (Angiomax ${ }^{\circledR}$, The Medicines Company, Parsippany, NJ) is a direct thrombin inhibitor (similar in activity to the direct thrombin inhibitors, hirudin and argatroban) and has been used in percutaneous coronary interventions. The major clinical utility of bivalirudin is for anticoagulation patients with a contraindication to heparin, specifically in patients with heparin-induced thrombocytopenia. ${ }^{1}$ Like heparin, the degree of anticoagulation is imperfectly followed using the aPTT. It should be noted that bivalirudin will also prolong the prothrombin time (PT) and international normalized ratio (INR). A typical dosing regimen for full anticoagulation (1.5 to 2.5 times baseline aPTT) is $0.7 \mathrm{mg} / \mathrm{kg}$ bolus with an infusion of $1.75 \mathrm{mg} / \mathrm{kg} / \mathrm{h}$. Bivalirudin has a plasma half-life of 25 minutes when administered IV, with $20 \%$ clearance by the kidneys. ${ }^{1}$ There is no specific reversal agent; however, factor concentrates (APTT; Feiba ${ }^{\circledR}$, Baxter Healthcare, One Baxter Parkway, Deerfield, IL) and recombinant factor VIIa (NovoSeven ${ }^{\circledR}$, Novo Nordisk, Inc., Princeton, NJ) induce partial reversal. ${ }^{8}$

\section{Warfarin (Coumadin ${ }^{\circledR}$ )}

Warfarin (Coumadin ${ }^{\circledR}$ ) is the most common vitamin $\mathrm{K}$ antagonist in use; it inhibits the procoagulant factors II, VII, IX, and $\mathrm{X}$ as well as the anticoagulant proteins $\mathrm{C}, \mathrm{S}$, and Z. ${ }^{9}$ Unlike heparins and bivalirudin, warfarin is administered orally. Warfarin is commonly used in the treatment of atrial fibrillation, deep venous thrombosis $(\mathrm{DVT}) /$ pulmonary embolism (PE), hypercoagulable 
states, and mechanical heart valves. Warfarin is best monitored using the INR, which is a standardized reporting value that utilizes an international standard to correct the PT into a value that is comparable and standardized between laboratories. ${ }^{10}$ Both the INR and PT evaluate the function of the extrinsic pathway of coagulation, evaluating warfarin inhibition of factors II, VII, and X. Of these, factor VII has the shortest half-life at 6 hours and is the cause of the earliest change of laboratory values.

The pharmacokinetics of warfarin are well understood and described elsewhere. ${ }^{9}$ Warfarin is water soluble, rapidly absorbed, and has high bioavailability. It reaches maximal blood concentration $\sim 90$ minutes after administration and circulates bound to albumin and other plasma proteins. It is degraded by the liver and has a half-life of 36 to 42 hours. ${ }^{9,11}$ Individual warfarin response is highly variable as the effects of warfarin are influenced by drug interactions, comorbidities, genetic variability, diet, nutritional supplements, and environmental factors. It should be noted that although the factor inhibition and anticoagulant effect may be evident after less than 48 hours of therapy, the full anticoagulant effect may take greater than 6 days to fully develop. ${ }^{9}$ Shortly after initiation of therapy, there is a period of relative hypercoagulability due to the reduction of vitamin K-dependent anticoagulant proteins. Delayed antithrombotic effect and transient hypercoagulability provide the rationale for heparinization when initiating treatment. A typical warfarin initiation regimen begins with LMWH or UFH and 5 or $10 \mathrm{mg}$ of warfarin daily with frequent INR checks. INR levels must be closely monitored until therapeutic and can eventually be spaced to monthly intervals. ${ }^{2}$

Reversal regimens for warfarin therapy (Table 2) range from simple withholding of the medication and a slow normalization of the INR to rapid reversal. The reversal regimen used is dictated by the nature of the procedure and concurrent patient factors. In patients who are at low risk for thrombotic complications and who need a temporary interruption of anticoagulation for a procedure, a 5 -day withdrawal is recommended. ${ }^{11}$ If the INR continues to be elevated ( $>1.5) 1$ to 2 days before the procedure, low-dose vitamin $\mathrm{K}$ oral administration ( $1 \mathrm{mg}$ for INR $1.5-1.9$ and $2 \mathrm{mg}$ for INR >1.9) is recommended by the American College of Chest Physicians (ACCP) guidelines. ${ }^{11}$

Reversal for patients at high risk of thrombotic or thromboembolic events (see comments below), such as recent venous thromboembolism or new atrial fibrillation, bridging anticoagulation with UFH (continuous IV infusion or $250 \mathrm{IU} / \mathrm{kg}$ subcutaneously twice daily) or LMWH (dalteparin $200 \mathrm{IU} / \mathrm{kg}$ daily; enoxaparin $1.5 \mathrm{mg} / \mathrm{kg}$; tinzaparin $175 \mathrm{IU} / \mathrm{kg}$ daily) administered subcutaneously is recommended. ${ }^{11}$ In patients with mechanical valves, IV UFH may be required for safe bridging therapy. Intravenous UFH in the therapeutic range (1.5-2 times the normal PTT) should be discontinued 4 hours before the procedure for complete elimination. ${ }^{11}$ However, in our practice we hold the heparin for at least 1 hour before starting the procedure. At therapeutic doses, this allows for approximately almost one half-life and active heparin will decrease for the duration of the procedure. For LMWH, the last dose should be administered 24 hours before the procedure is scheduled. ${ }^{11}$

Urgent reversal of warfarin can be performed with the administration of low-dose vitamin $\mathrm{K}(2.5-5 \mathrm{mg}) .^{11}$ Oral administration of vitamin $\mathrm{K}$ is preferred over parenteral routes. ${ }^{9}$ Larger doses should be avoided, as patients treated with large doses of vitamin $\mathrm{K}$ can become resistant to warfarin due to vitamin $\mathrm{K}$ accumulation in the liver for up to one week. ${ }^{9}$ If the INR is in the therapeutic range, emergent reversal of warfarin is accomplished by the transfusion of fresh frozen plasma (FFP) and vitamin $\mathrm{K}$ (10 $\mathrm{mg}$ if bleeding; $2.5-5 \mathrm{mg}$ for emergent procedure). ${ }^{9}$ If the INR is supratherapeutic, the amount of FFP required to correct the coagulopathy may be considerable, and will necessitate a prolonged infusion. In this situation, the use of factor concentrates (APTT; Feiba ${ }^{\mathbb{R}}$ ) or recombinant factor VIIa $\left(\right.$ NovoSeven ${ }^{\circledR}$ ) have been described. ${ }^{9}$

Table 2 Time-Adjusted Reversal of Warfarin*

\begin{tabular}{ll}
\hline Time until Procedure & Intervention for Reversal \\
\hline Greater than 5 days & Hold warfarin 5 days before procedure \\
5 days to 24 hours & Hold warfarin and check INR 24 hours preprocedure \\
24 hours & Hold warfarin, check INR \\
& - For INR 1.5-1.9 give $1 \mathrm{mg}$ vitamin K orally \\
& - For INR $>1.9$ give $2 \mathrm{mg}$ vitamin K orally \\
Less than 24 hours or emergent & Hold warfarin, check INR \\
& - For INR 1.5-3 give 2 units fresh frozen plasma and recheck INR \\
& - For INR $>3$ give fresh frozen plasma and consider factor concentrates
\end{tabular}

*Ideally, reversal of warfarin is planned prior to the procedure. If recognized long in advance of the procedure, warfarin may simply be withheld. However, if the procedure is emergent, reversal with fresh frozen plasma or factor concentrates may be required to safely reverse warfarin. INR, International normalized ratio. 
Resuming anticoagulant therapy is also predicated on individual patient factors and procedural risk. If the procedure has a low risk for bleeding, LMWH and the patient's previous dosing regimen of warfarin should be resumed 12 to 24 hours postprocedure and continued until the INR is therapeutic. ${ }^{11}$ Resuming anticoagulation should be delayed if the procedure places the patient at an increased risk of bleeding with a low risk of thrombotic or thromboembolic complications. As bleeding complications of outpatient procedures often occur outside the recovery area, special consideration of patient factors merits assessment before resuming anticoagulation. Delaying resumption of LMWH 24 to 72 hours postprocedure, as well as using a lower dose regimen (enoxaparin $30 \mathrm{mg}$ twice daily; dalteparin 5,000 IU daily) are described strategies for reducing bleeding risk after high-risk procedures or in patients with increased bleeding risk for other reasons. ${ }^{11}$

\section{PROCEDURAL RISK}

Procedural bleeding risk (Fig. 1) is subjective and represents a composite risk based on the procedure being performed, patient factors, and operator experience. An excellent assessment of procedural risk is addressed in the Society of Interventional Radiology Consensus Guidelines. ${ }^{10}$ As a summary, procedures void of other factors may be classified into low risk, moderate risk, and significant risk. Low-risk procedures include nontunneled venous access devices, drainage catheter exchange, and superficial biopsies. Moderate risk procedures include vascular interventions, chemoembolization, tunneled venous access devices, deep biopsies, and gastrostomy tube placement. Procedures considered to have a significant risk of bleeding include renal biopsy, nephrostomy tube placement, and transjugular intrahepatic portosystemic shunt (TIPS) placement. An important component of procedural risk is assessment of potential complications. Lesions that are close to vital structures have an increased risk for bleeding overall. With imageguided intervention the ability to recognize significant bleeding is limited, and controlling deep bleeding may be difficult.

Furthermore, the overall risk of performing (and successfully recovering from) a procedure is also affected by patient factors and postprocedural considerations. Other factors that place patients at increased risk may include other medications that affect hemostasis (e.g., antiplatelet agents), obesity, comorbidities, patient motion, critical adjacent structures, and associated anatomy. Postprocedural considerations also apply; these risk factors include the ability to monitor the patient postprocedure, the need to start or restart anticoagulation, and the patient's psychosocial situation that might preclude his or her ability to recognize or respond appropriately to complications after an outpatient procedure. For exam- ple, a "simple" biopsy will have increased risk in an obese diabetic with questionable aptitude who lives alone and remote from medical care.

Equipment limitations also may fall under this category, although these are difficult to quantify. High-resolution versus low-resolution ultrasound for visualization, or being unfamiliar with the equipment available, would increase the bleeding risk of the procedure, potentially from multiple passes or a suboptimal approach.

A final important consideration is the experience of the operator and team. This applies in two broad senses. First, experience decreases the overall procedural time and complication rate. Second, experience also assists in the assessment of procedural risk and patient risk.

\section{PATIENT RISK OFF ANTICOAGULATION THERAPY}

Stopping or reversing anticoagulation therapy is not without risk and there may be transient rebound hypercoagulability from the cessation and subsequent resumption of anticoagulation therapy. Therefore, the risk of anticoagulation therapy cessation also needs stratification. This is based on the indication for anticoagulation therapy, and can be categorized into low or high risk based on indication and length of therapy. Common indications for anticoagulation therapy are addressed here; however, it should be noted that iterations of indications and confounding factors are infinite.

Atrial fibrillation increases the risk of thromboembolism from a cardiac source. However, patients with atrial fibrillation are not a homogenous group. Patients with atrial fibrillation on less than one month of anticoagulation therapy are at increased risk from theoretic residual atrial thrombus, whereas patients that have been treated for greater than one month are likely to have had time to resolve any atrial thrombus burden. ${ }^{12}$ Patients with atrial fibrillation have been stratified for embolic risk based on many classifications. The following risk factors have been identified: history of prior stroke, age $>65$ years, diabetes mellitus, congestive heart failure, and hypertension. ${ }^{13,14}$ The risk of stroke in patients without these risk factors is low, and temporary cessation of anticoagulation therapy carries a low risk. In patients with all of the risk factors, the risk of an event is still low, with an annual risk of less than $15 \% .{ }^{15}$ Therefore, in patients treated for atrial fibrillation for longer than one month, no bridging therapy is required. Bridging therapy may be considered in patients at high risk by the aforementioned clinical criteria, but even in high-risk patients the risk of short-term anticoagulation therapy cessation is relatively low. Patients with anticoagulation therapy of less than one-month duration and unknown atrial thrombus status (by echocardiography) 


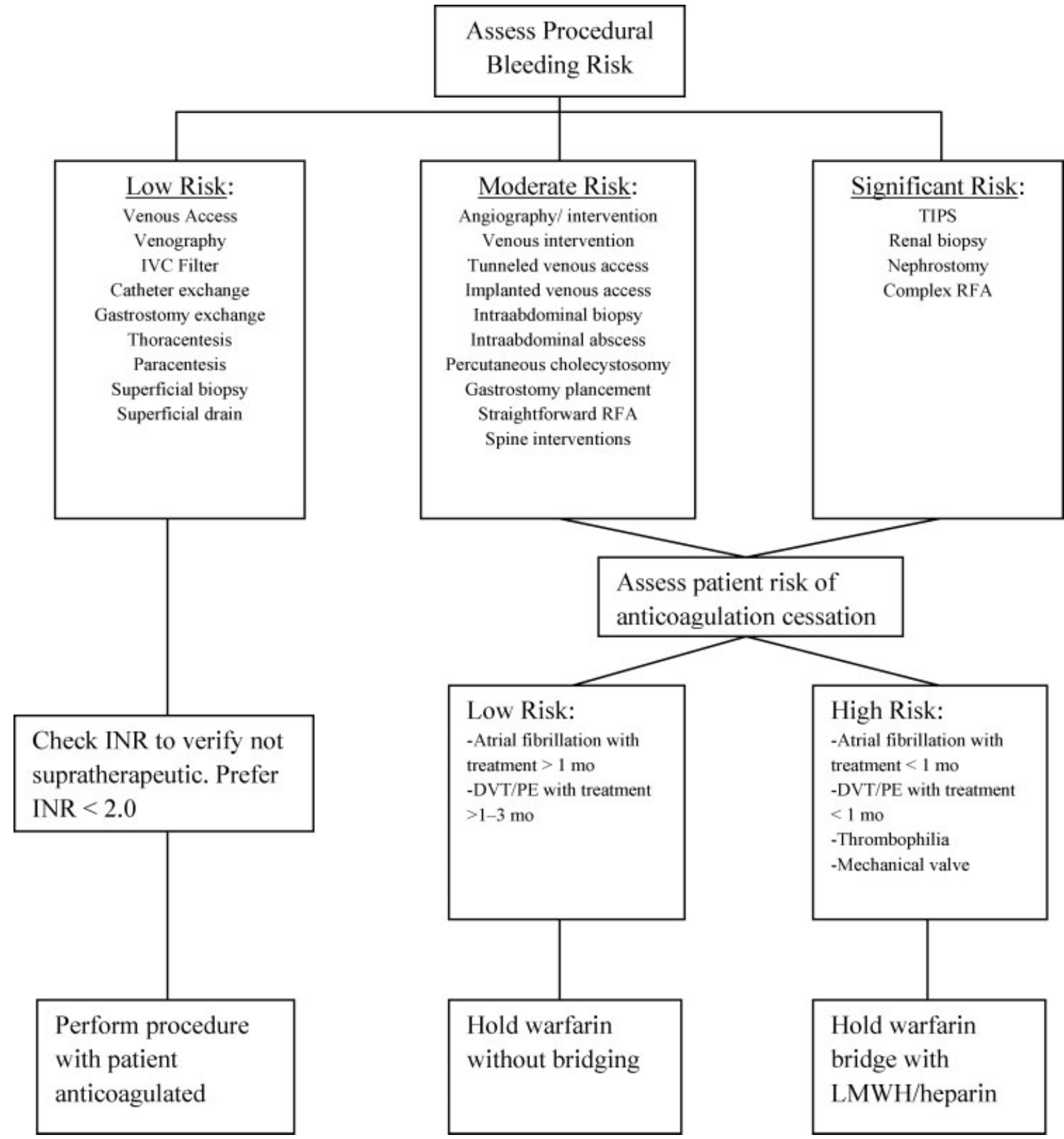

Figure 1 Preprocedure approach to the anticoagulated patient. Before a routine or emergent procedure a risk assessment is performed. For low bleeding risk procedures, the procedure may be performed with the patient anticoagulated. For moderate and significant risk categories, an additional assessment of the risk of anticoagulation cessation is required. TIPS, transjugular intrahepatic portosystemic shunts; INR, international normalized ratio; DVT, deep venous thrombosis; PE, pulmonary embolism; LMWH, low-molecular-weight heparin; RFA, radiofrequency ablation; IVC, inferior vena cava.

should be considered high risk for cessation of anticoagulation. If the procedure cannot be delayed, bridging therapy should be administered.

Pulmonary embolism and venous thromboembolic disease are other common indications for anticoagulation therapy. Similar to atrial fibrillation, the length of therapy is a key determinant of cessation risk. In patients who have been treated for one month (and ideally 3 months) the risk of recurrent disease with temporary cessation is low. ${ }^{16}$ If the patient is within one month of an acute PE or DVT and the procedure cannot be delayed, bridging therapy should be considered.

Mechanical valves create a unique problem. All patients with mechanical valves require anticoagulation therapy. ${ }^{17}$ Patients with any mitral valve replacement or a mechanical aortic valve replacement requiring anticoagulation cessation should hold warfarin and be bridged with UFH when the INR falls below 2.0. ${ }^{17}$ 
The heparin may then be stopped 1 to 4 hours before the procedure if complete anticoagulation reversal is required. Patients with a bileaflet mechanical aortic valve replacement may be an exception to this rule, depending on risk factors, and consultation with the patient's cardiologist is recommended.

Hypercoagulable states, inherited and acquired, also increase the risk of severe thrombotic complication if anticoagulation is withheld or reversed. With regard to the inherited thrombophilias, with the possible exception of the factor $\mathrm{V}$ Leiden gene mutation all patients should be considered at high risk for cessation of anticoagulation therapy and be bridged appropriately with LMWH or UFH. ${ }^{18}$ As a general guideline, if the patient requires lifelong anticoagulation for a hypercoagulable state, the risk of cessation is high and the patient should be bridged. Consultation with the referring physician and/or the patient's hematologist is recommended. Acquired hypercoagulable states due to neoplastic disease represent a heterogenous population. Routine prophylaxis of cancer patients is generally not recommended, and if the patient is on anticoagulation this may be an indicator of complicating concurrent therapy or thrombotic complication that may require bridging therapy. ${ }^{19}$ Consultation with the patient's oncologist is recommended in this unique situation.

\section{SUMMARY}

Medical management is required of providers performing invasive procedures. To fully assess the requirement of anticoagulation cessation or reversal, both the procedure-associated risk and the risk to the patient must be assessed. Procedures with low bleeding risk may not require withholding or the reversal of anticoagulation therapy. Conversely, procedures with high bleeding risk will commonly require reversal of anticoagulation therapy.

However, consideration of the patient's risk off anticoagulation therapy is necessary. Patients at low risk for thromboembolic events, such as patients treated for atrial fibrillation or DVT for a long duration may simply hold their warfarin prior to the procedure. Patients that are at high risk for thromboembolic events, such as patients with mechanical heart valves, require bridging therapy up to the procedure time to minimize the risk of periprocedural thromboembolic events.

Image-guided procedures can carry additional risk compared with open procedures because the ability to detect and control bleeding is reduced. Optimizing a patient's coagulation profile prior to an invasive procedure is a mandatory component of the preprocedure work-up. A systematic approach to patients who require anticoagulation is vital and should improve outcomes in this specific patient population.

\section{REFERENCES}

1. Hirsh J, Bauer KA, Donati MB, Gould M, Samama MM, Weitz JI; American College of Chest Physicians. Parenteral anticoagulants: American College of Chest Physicians Evidence-Based Clinical Practice Guidelines (8th Edition) Chest 2008;133(6, Suppl):141S-159S

2. du Breuil AL, Umland EM. Outpatient management of anticoagulation therapy. Am Fam Physician 2007;75(7): 1031-1042

3. Raschke RA, Reilly BM, Guidry JR, Fontana JR, Srinivas S. The weight-based heparin dosing nomogram compared with a "standard care" nomogram. A randomized controlled trial. Ann Intern Med 1993;119(9):874-881

4. Kubalek R, Berlis A, Schwab M, Klisch J, Schumacher M. Activated clotting time or activated partial thromboplastin time as the method of choice for patients undergoing neuroradiological intervention. Neuroradiology 2003;45(5): 325-327

5. Siskin GP, Reiner E, Stainken BF, et al. Activated clotting time as a screening test prior to catheter-based cardiovascular procedures. Catheter Cardiovasc Interv 2001;54(2):191-195

6. Shah MaSJeanne P. Heparin-induced thrombocytopenia occurring after discontinuation of heparin. Medscape Radiology. Available at: http://www.medscape.com/viewarticle/ 452276. Accessed April 23, 2003

7. Rajgopal R, Bear M, Butcher MK, Shaughnessy SG. The effects of heparin and low molecular weight heparins on bone. Thromb Res 2008;122(3):293-298

8. Sørensen B, Ingerslev J. A direct thrombin inhibitor studied by dynamic whole blood clot formation. Haemostatic response to ex-vivo addition of recombinant factor VIIa or activated prothrombin complex concentrate. Thromb Haemost 2006;96(4):446-453

9. Ansell J, Hirsh J, Hylek E, Jacobson A, Crowther M, Palareti G; American College of Chest Physicians. Pharmacology and management of the vitamin $\mathrm{K}$ antagonists: American College of Chest Physicians Evidence-Based Clinical Practice Guidelines (8th Edition). Chest 2008;133(6, Suppl):160S-198S

10. Malloy PC, Grassi CJ, Kundu S, et al; Standards of Practice Committee with Cardiovascular and Interventional Radiological Society of Europe (CIRSE) Endorsement. Consensus guidelines for periprocedural management of coagulation status and hemostasis risk in percutaneous image-guided interventions. J Vasc Interv Radiol 2009;20(7, Suppl):S240 S249

11. Douketis JD, Berger PB, Dunn AS, et al; American College of Chest Physicians. The perioperative management of antithrombotic therapy: American College of Chest Physicians Evidence-Based Clinical Practice Guidelines (8th Edition). Chest 2008;133(6, Suppl):299S-339S

12. Collins LJ, Silverman DI, Douglas PS, Manning WJ. Cardioversion of nonrheumatic atrial fibrillation. Reduced thromboembolic complications with 4 weeks of precardioversion anticoagulation are related to atrial thrombus resolution. Circulation 1995;92(2):160-163

13. Gage BF, Waterman AD, Shannon W, Boechler M, Rich MW, Radford MJ. Validation of clinical classification schemes for predicting stroke: results from the National Registry of Atrial Fibrillation. JAMA 2001;285(22):2864-2870

14. Go AS, Hylek EM, Chang Y, et al. Anticoagulation therapy for stroke prevention in atrial fibrillation: how well do randomized trials translate into clinical practice? JAMA 2003;290(20):2685-2692 
15. Singer DE, Albers GW, Dalen JE, et al; American College of Chest Physicians. Antithrombotic therapy in atrial fibrillation: American College of Chest Physicians EvidenceBased Clinical Practice Guidelines (8th Edition). Chest 2008;133(6, Suppl):546S-592S

16. Levine MN, Hirsh J, Gent M, et al. Optimal duration of oral anticoagulant therapy: a randomized trial comparing four weeks with three months of warfarin in patients with proximal deep vein thrombosis. Thromb Haemost 1995;74(2): 606-611

17. Bonow RO, Carabello BA, Chatterjee $\mathrm{K}$, et al; American College of Cardiology; American Heart Association Task Force on Practice Guidelines (Writing Committee to Revise the 1998 Guidelines for the Management of Patients with Valvular Heart Disease); Society of Cardiovascular Anesthesiologists. ACC/AHA 2006 Guidelines for the Management of Patients with Valvular Heart Disease: A Report of the American College of Cardiology/American Heart Association Task Force on Practice Guidelines (Writing Committee to Revise the 1998 Guidelines for the Management of Patients with Valvular Heart Disease) Developed in Collaboration with the Society of Cardiovascular Anesthesiologists Endorsed by the Society for Cardiovascular Angiography and Interventions and the Society of Thoracic Surgeons. J Am Coll Cardiol 2006;48(3):e1-e148

18. Martinelli I, Mannucci PM, De Stefano V, et al. Different risks of thrombosis in four coagulation defects associated with inherited thrombophilia: a study of 150 families. Blood 1998;92(7):2353-2358

19. Lyman GH, Khorana AA, Falanga A, et al; American Society of Clinical Oncology. American Society of Clinical Oncology guideline: recommendations for venous thromboembolism prophylaxis and treatment in patients with cancer. J Clin Oncol 2007;25(34):5490-5505 